\title{
Semiholonomic Second Order Connections Associated with Material Bodies
}

\author{
Jaroslav Hrdina and Petr Vašík \\ Institute of Mathematics, Faculty of Mechanical Engineering, Brno University of Technology, Technická 2, 61669 Brno, Czech Republic \\ Correspondence should be addressed to Petr Vašík; vasik@fme.vutbr.cz
}

Received 24 August 2012; Revised 12 November 2012; Accepted 13 November 2012

Academic Editor: Baolin Wang

Copyright (C) 2013 J. Hrdina and P. Vašík. This is an open access article distributed under the Creative Commons Attribution License, which permits unrestricted use, distribution, and reproduction in any medium, provided the original work is properly cited.

The thermomechanical behavior of a material is expressed mathematically by means of one or more constitutive equations representing the response of the body to the history of its deformation and temperature. These settings induce a set of connections which can express local properties. We replace two of them by a second order connection and prove that the holonomity of this connection classifies our materials.

\section{Introduction}

The use of differential geometry in material science is based on 1-jet calculus. This technique is described in, for example, $[1,2]$. A material body endowed with a constitutional equation induces naturally a linear connection, and several important physical properties of the material are described by means of its geodesics. The cited books handle one constitutional equation and thus one appropriate linear connection. In case that a material is endowed with more than one constitution equation, that is, by more than one connection, the topic of higher order connections appears. Note that the topic of higher order connections is widely studied; see, for example, [3-5]. Such approach is not established so far in the material science, and this paper thus formulates introductory principles and problems of the theory of materials endowed with more than one constitution equation.

\section{Geometric Motivation of Higher Order Connections}

To show the compatibility with the geometric concept of a connection, let us now recall its generalization to higher order connections; see [6] for general concepts. The following section is based on [7].

Definition 1. A connection on bundle $\pi: M_{1} \rightarrow M$ is defined by the structure $\Delta_{h} \oplus \Delta_{v}$ on a manifold $M_{1}$ where $\Delta_{v}=\operatorname{ker} T \pi$ is vertical distribution tangent to the fibers and $\Delta_{h}$ is horizontal distribution complementary to the distribution $\Delta_{v}$. The transport of the fibers along the path $\gamma \mathrm{C}$ $M$ is realized by the horizontal lifts given by the distribution $\Delta_{h}$ on the surface $\pi^{-1}(\gamma)$. If the bundle is a vector one and the transport of fibers along an arbitrary path is linear, then the connection is called linear.

We will assume that the base manifold $M$ is of dimension $n$ and the fibers are of dimension $r$. Then

$$
\operatorname{dim} \Delta_{h}=n, \quad \operatorname{dim} \Delta_{v}=r .
$$

On the neighborhood $U \subset M_{1}$, let us consider local base and fiber coordinates:

$$
\left(u^{i}, u^{\alpha}\right), \quad i=1,2, \ldots, n ; \alpha=n+1, \ldots, n+r .
$$

Base coordinates $\left(u^{i}\right)$ are determined by the projection $\pi$ and the coordinates $\left(\bar{u}^{i}\right)$ on a neighborhood $\bar{U}=\pi(U), u^{i}=\bar{u}^{i} \circ \pi$.

Definition 2. On a neighborhood $U \subset M_{1}$ we define a local (adapted) basis of the structure $\Delta_{h} \oplus \Delta_{v}$ :

$$
\begin{aligned}
\left(X_{i} X_{\alpha}\right) & =\left(\frac{\partial}{\partial u^{j}} \frac{\partial}{\partial u^{\beta}}\right) \cdot\left(\begin{array}{cc}
\delta_{i}^{j} & 0 \\
\Gamma_{i}^{\beta} & \delta_{\alpha}^{\beta}
\end{array}\right), \\
\left(\begin{array}{c}
\omega^{i} \\
\omega^{\alpha}
\end{array}\right) & =\left(\begin{array}{cc}
\delta_{j}^{i} & 0 \\
-\Gamma_{j}^{\alpha} & \delta_{\beta}^{\alpha}
\end{array}\right) \cdot\left(\begin{array}{l}
d u^{j} \\
d u^{\beta}
\end{array}\right) .
\end{aligned}
$$


The horizontal distribution $\Delta_{h}$ is the linear span of the vector fields $\left(X_{i}\right)$ and the annihilator of the forms $\left(\omega^{\alpha}\right)$,

$$
X_{i}=\partial_{i}+\Gamma_{i}^{\beta} \partial_{\beta}, \quad \omega^{\alpha}=d u^{\alpha}-\Gamma_{i}^{\alpha} d u^{i} .
$$

Definition 3. A classical affine connection on manifold $M$ is seen as a linear connection on the bundle $\pi_{1}: T M \rightarrow M$. On the tangent bundle $T M \rightarrow M$ one can define the structure $\Delta_{h} \oplus \Delta_{v}$. The indices in the formulas are denoted by Latin letters all of them ranging from 1 to $n$. The functions $\Gamma_{i}^{\alpha}, X_{i}$, and $\omega^{\alpha}$ are of the form (in $\Gamma_{i}^{\alpha}$ the sign is changed to comply with the classical theory):

$$
\begin{gathered}
\Gamma_{i}^{\alpha} \rightsquigarrow-\Gamma_{j k}^{i} u_{1}^{k}, \\
X_{i}=\partial_{i}+\Gamma_{i}^{\alpha} \partial_{\alpha} \rightsquigarrow X_{i}=\partial_{i}-\Gamma_{i j}^{k} u_{1}^{i} \partial_{k}^{1}, \\
\omega^{\alpha}=d u^{\alpha}-\Gamma_{i}^{\alpha} d u^{i} \leadsto U_{12}^{i}=u_{12}^{i}+\Gamma_{j k}^{i} u_{1}^{k} u_{2}^{j} .
\end{gathered}
$$

Definition 4. Higher order connections are defined as follows: on tangent bundle $T M$ the structure $\Delta \oplus \Delta_{1}$ is defined where $\operatorname{ker} T \rho_{1}=\Delta_{1}$, on $T(T M)$ the structure $\Delta \oplus \Delta_{1} \oplus \Delta_{2} \oplus \Delta_{12}$ is defined where $\operatorname{ker} T \rho_{s}=\Delta_{s} \oplus \Delta_{12}, s=1,2$, and so forth.

\section{Jet Prolongation of a Fibered Manifold and Higher Order Connections}

To compute with second order connections in an efficient way we have to go deeper in the theory. Structural approach introduced by C. Ehresmann and developed in, for example, [3] reads that $r$ th order holonomic prolongation $J^{r} Y$ of $Y$ is a space of $r$-jets of local sections $M \rightarrow Y$ and nonholonomic prolongation $\widetilde{J}^{r} Y$ of $Y$ is defined by the following iteration:

(1) $\widetilde{J}^{1} Y=J^{1} Y$; that is, $\widetilde{J}^{1} Y$ is a space of 1 -jets of sections $M \rightarrow Y$ over the target space $Y$;

(2) $\widetilde{J}^{r} Y=J^{1}\left(\widetilde{J}^{r-1} Y \rightarrow M\right)$.

Clearly, we have an inclusion $J^{r} Y \subset \widetilde{J}^{r} Y$ given by $j_{x}^{r} \gamma \mapsto$ $j_{x}^{1}\left(j^{r-1} \gamma\right)$. Further, $r$ th semiholonomic prolongation $\bar{J}^{r} Y \subset$ $\widetilde{J}^{r} Y$ is defined by the following induction. First, by $\beta_{1}=\beta_{Y}$ we denote the projection $J^{1} Y \rightarrow Y$ and by $\beta_{r}=\beta_{\widetilde{J}^{r-1} Y}$ the projection $\widetilde{J}^{r} Y=J^{1} \widetilde{J}^{r-1} Y \rightarrow \widetilde{J}^{r-1} Y, r=2,3, \ldots$ If we set $\bar{J}^{1} Y=J^{1} Y$ and assume we have $\bar{J}^{r-1} Y \subset \widetilde{J}^{r-1} Y$ such that the restriction of the projection $\beta_{r-1}: \widetilde{J}^{r-1} Y \rightarrow \widetilde{J}^{r-2} Y$ maps $\bar{J}^{r-1} Y$ into $\bar{J}^{r-2} Y$, we can construct $J^{1} \beta_{r-1}: J^{1} \bar{J}^{r-1} Y \rightarrow$ $J^{1} \bar{J}^{r-2} Y$ and define

$$
\bar{J}^{r} Y=\left\{A \in J^{1} \bar{J}^{r-1} Y ; \beta_{r}(A)=J^{1} \beta_{r-1}(A) \in \bar{J}^{r-1} Y\right\} .
$$

Obviously, $J^{r}, \bar{J}^{r}$, and $\widetilde{J}^{r}$ are bundle functors on the category $\mathscr{F} \mathscr{M}_{m, n}$ of fibered manifolds with $m$-dimensional bases and $n$-dimensional fibres and locally invertible fiber-preserving mappings.

Alternatively, one can define the $r$ th order semiholonomic prolongation $\bar{J}^{r} Y$ by means of natural target projections of nonholonomic jets; see [4]. For $r \geq q \geq 0$ let us denote by $\pi_{q}^{r}$ the target surjection $\pi_{q}^{r}: \widetilde{J}^{r} Y \rightarrow \widetilde{J}^{q} Y$ with $\pi_{r}^{r}$ being the identity on $\widetilde{J}^{r} Y$. We note that the restriction of these projections to the subspace of semiholonomic jet prolongations will be denoted by the same symbol. By applying the functor $J^{k}$ we have also the surjections $J^{k} \pi_{q-k}^{r-k}$ : $\widetilde{J}^{r} Y \rightarrow \widetilde{J}^{q} Y$, and, consequently, the element $X \in \widetilde{J}^{r} Y$ is semiholonomic if and only if

$$
\left(J^{k} \pi_{q-k}^{r-k}\right)(X)=\pi_{q}^{r}(X) \text { for any integers } 1 \leq k \leq q \leq r .
$$

In [4], the proof of this property can be found and the author finds it useful when handling semiholonomic connections and their prolongations.

Finally, the following functorial definition of semiholonomic prolongation of a fibered manifold can be found. Assume that the functor $\bar{J}^{r-1}$ comes equipped with the canonical transformation $\bar{J}^{r-1} \rightarrow \bar{J}^{r-2}$ given by the restriction of jet target projections. Then there are two canonical transformations $J^{1} \bar{J}^{r-1} \rightarrow J^{1} \bar{J}^{r-2}$, and one can define $\bar{J}^{r}$ as the equalizer of these two transformations. Then this is equivalent to the definition

$$
\bar{J}^{r} Y=\bar{J}^{2}\left(\bar{J}^{r-2} Y\right) \cap J^{1}\left(\bar{J}^{r-1} Y\right) .
$$

To define a higher order connection we start with the definition of general connection; see [3].

Definition 5. A general connection on the fibered manifold $Y \rightarrow M$ is a section $\Gamma: Y \rightarrow J^{1} Y$ of the first jet prolongation $J^{1} Y \rightarrow Y$.

By the substitution of the target space by $J^{r} Y, \bar{J}^{r} Y$, and $\widetilde{J}^{r} Y$, respectively, one obtains definition of $r$ th order holonomic, semiholonomic, and nonholonomic connections; that is, a higher order connection is a section of the appropriate jet prolongation of a fibered manifold.

Let us recall that the semiholonomity condition on a higher order connection defined in the geometric way is now transformed into the equality of all projections $T \rho_{s}$ from Definition 4.

Previous approach to connections is suitable for conceptual considerations and operations with connections, such as prolongations of connections, natural operators, and some classifications. For us the following theorem is quite useful; see [8] for the proof.

Theorem 6. (1) Second order nonholonomic connections $\theta$ : $Y \rightarrow \widetilde{J}^{2} Y$ on $Y \rightarrow M$ are in bijection with triples $\left(\Gamma_{1}, \Gamma_{2}, G\right)$, where $\Gamma_{1}, \Gamma_{2}: Y \rightarrow J^{1} Y$ are first order connections on $Y \rightarrow M$ and $G: Y \rightarrow V Y \otimes \otimes^{2} T^{*} M$ is a tensor field. (2) Connection $\theta$ is semiholonomic if and only if $\Gamma_{1}=\Gamma_{2}$. (3) Connection $\theta$ is holonomic if and only if $\Gamma_{1}=\Gamma_{2}$ and $G: Y \rightarrow V Y \otimes S^{2} T^{*} M$.

Now, one can define the following relation $\sim$ on the space of second order nonholonomic connections.

Definition 7. Let the triples $\left(\Gamma_{1}, \Gamma_{2}, G\right),\left(\bar{\Gamma}_{1}, \bar{\Gamma}_{2}, \bar{G}\right)$ represent two second order connections in the sense of Theorem 6 . 
They belong to the same equivalence class of the relation if and only if $\Gamma_{1}=\bar{\Gamma}_{1}$ and $\Gamma_{2}=\bar{\Gamma}_{2}$.

Remark 8. It is easy to see that $\sim$ is an equivalence relation and let us denote $[\theta]=\left[\left(\Gamma_{1}, \Gamma_{2}, G\right)\right]$ as the a class of this relation. Finally, the class $[\theta]$ consists of semiholonomic connections if and only if $\Gamma_{1}=\Gamma_{2}$ for any $\left(\Gamma_{1}, \Gamma_{2}, G\right) \in[\theta]$ and holonomic if $G: Y \rightarrow V Y \otimes S^{2} T^{*} M$ in addition.

\section{Ehresmann Prolongation}

Given two higher order connections $\Gamma: Y \rightarrow \widetilde{J}^{r} Y$ and $\bar{\Gamma}: Y \rightarrow \widetilde{J}^{s} Y$, the product of $\Gamma$ and $\bar{\Gamma}$ is the $(r+s)$ th order connection $\Gamma * \bar{\Gamma}: Y \rightarrow \widetilde{J}^{r+s} Y$ defined by

$$
\Gamma * \bar{\Gamma}=\widetilde{J}^{s} \Gamma \circ \bar{\Gamma} .
$$

Concerning the holonomity, according to [4] if both $\Gamma$ and $\bar{\Gamma}$ are of the first order, then $\Gamma * \bar{\Gamma}: Y \rightarrow \widetilde{J}^{2} Y$ is semiholonomic if and only if $\Gamma=\bar{\Gamma}$ and $\Gamma * \bar{\Gamma}$ is holonomic if and only if $\Gamma$ is curvature-free in addition, which corresponds to Theorem 6 .

As an example we show the coordinate expression of an arbitrary nonholonomic second order connection and of the product of two first order connections. The coordinate form of $\Delta: Y \rightarrow \widetilde{J}^{2} Y$ is

$$
y_{i}^{p}=F_{i}^{p}(x, y), \quad y_{0 i}^{p}=G_{i}^{p}(x, y), \quad y_{i j}^{p}=H_{i j}^{p}(x, y),
$$

where $F, G$, and $H$ are arbitrary smooth functions. Further, if the coordinate expressions of two first order connections $\Gamma, \bar{\Gamma}: Y \rightarrow J^{1} Y$ are

$$
\Gamma: y_{i}^{p}=F_{i}^{p}(x, y), \quad \bar{\Gamma}: y_{i}^{p}=G_{i}^{p}(x, y),
$$

then the second order connection $\Gamma * \bar{\Gamma}: Y \rightarrow \widetilde{J}^{2} Y$ has equations

$$
y_{i}^{p}=F_{i}^{p}, \quad y_{0 i}^{p}=G_{i}^{p}, \quad y_{i j}^{p}=\frac{\partial F_{i}^{p}}{\partial x^{j}}+\frac{\partial F_{i}^{p}}{\partial y^{q}} G_{j}^{q} .
$$

For order three see [5]. If we apply the multiplication on just one connection $\Gamma$, the second order connection $\Gamma * \Gamma$ is called the Ehresmann prolongation of connection $\Gamma$. By iteration we obtain a connection of an arbitrary order.

In the following proposition we show that concerning order 2 only the choice of Ehresmann prolongation makes sense. We use the notation of [3], where the map e $: \bar{J}^{2} Y \rightarrow$ $\bar{J}^{2} Y$ is obtained from the natural exchange map $e_{\Lambda}: J^{1} J^{1} Y \rightarrow$ $J^{1} J^{1} Y$ as a restriction to the subbundle $\bar{J}^{2} Y \subset J^{1} J^{1} Y$. Note that while $e_{\Lambda}$ depends on the linear connection $\Lambda$ on $M$, its restriction $e$ is independent of any auxiliary connections. We remark that originally the map $e_{\Lambda}$ was introduced by $\mathrm{M}$. Modugno. We also recall that J. Pradines introduced a natural map $\bar{J}^{2} Y \rightarrow \bar{J}^{2} Y$ with the same coordinate expression.

Now we are ready to recall the following assertion; see [9] for the proof.
Proposition 9. All natural operators transforming first order connection $\Gamma: Y \rightarrow J^{1} Y$ into second order semiholonomic connection $Y \rightarrow \bar{J}^{2} Y$ form a one-parameter family:

$$
\Gamma \longmapsto k \cdot(\Gamma * \Gamma)+(1-k) \cdot e(\Gamma * \Gamma), \quad k \in \mathbb{R} .
$$

To meet the classical theory mentioned in Section 2 let us note that the corresponding operation is the following; see also [10]. If we apply the tangent functor $T$ two times on a projection $\pi: E \rightarrow M$ and a section $\sigma: M \rightarrow E$ we obtain

$$
\begin{array}{ll}
T \pi: T E \longrightarrow T M, & T^{2} \pi: T^{2} E \longrightarrow T^{2} M, \\
T \sigma: T M \longrightarrow T E, & T^{2} \sigma: T^{2} M \longrightarrow T^{2} E,
\end{array}
$$

respectively. The mappings $\sigma, T \sigma$, and $T^{2} \sigma$ are defined by the sections of fibered manifolds $\pi, T \pi$, and $T^{2} \pi$.

Let us consider local coordinates on the following manifolds in the form

$$
\begin{aligned}
& \text { on } M, T M, T^{2} M:\left(x^{i}\right),\left(x^{i}, x_{1}^{i}\right),\left(x^{i}, x_{1}^{i}, x_{2}^{i}, x_{12}^{i}\right) \text {, } \\
& \text { on } E, T E, T^{2} E: \quad\left(y^{p}\right),\left(y^{p}, y_{1}^{p}\right),\left(y^{p}, y_{1}^{p}, y_{2}^{p}, y_{12}^{p}\right) .
\end{aligned}
$$

Let us also consider a function $f$ defined on a manifold $M$, whose local coordinate form is derived by means of differentials to fit the coordinates on $T^{2} M$ :

$$
f_{1} \doteq f_{i} x_{1}^{i}, \quad f_{2} \doteq f_{i} x_{2}^{i}, \quad f_{12} \doteq f_{i j} x_{1}^{i} x_{2}^{j}+f_{i} x_{12}^{i},
$$

$$
\text { where } f_{i}=\frac{\partial f}{\partial x^{i}}, \quad f_{i j}=\frac{\partial^{2} f}{\partial x^{i} \partial x^{j}} \text {. }
$$

Furthermore, $f_{1}=\mathrm{d} f \circ \rho_{1}, f_{2}=\mathrm{d} f \circ \rho_{2}, f_{12}=\mathrm{d}^{2} f$. We use this notation in the following formulae.

If the section $\sigma$ is defined by local functions $\Gamma^{p}$, then the sections $T \sigma$ and $T^{2} \sigma$ are defined by its differentials $\Gamma_{1}^{p}, \Gamma_{2}^{p}$, and $\Gamma_{12}^{p}$ :

$$
\begin{aligned}
& \sigma: x^{i} \rightsquigarrow y^{p}=\Gamma^{p}, \\
& T \sigma:\left(x^{i}, x_{1}^{i}\right) \rightsquigarrow\left(y^{p}, y_{1}^{p}\right)=\left(\Gamma^{p}, \Gamma_{1}^{p}\right), \\
& T^{2} \sigma:\left(x^{i}, x_{1}^{i}, x_{2}^{i}, x_{12}^{i}\right) \rightsquigarrow\left(y^{p}, y_{1}^{p}, y_{2}^{p}, y_{12}^{p}\right)=\left(\Gamma^{p}, \Gamma_{1}^{p}, \Gamma_{2}^{p}, \Gamma_{12}^{p}\right),
\end{aligned}
$$$$
\text { where } \Gamma_{1}^{p}=\Gamma_{i}^{p} x_{1}^{i}, \quad \Gamma_{2}^{p}=\Gamma_{i}^{p} x_{2}^{i}, \quad \Gamma_{12}^{p}=\Gamma_{i j}^{p} x_{1}^{i} x_{2}^{j}+\Gamma_{i}^{p} x_{12}^{i} \text {. }
$$

The case when the coefficients $\Gamma_{i}^{p}$ and $\Gamma_{i j}^{p}$ in (18) are arbitrary functions corresponds to a nonholonomic connection on the fibered manifold $\pi$.

The case when $\Gamma_{i j}^{p}=\partial \Gamma_{i}^{p} / \partial x^{j}$, where $\Gamma_{i}^{p}$ are arbitrary functions corresponds to a semiholonomic connection on the fibered manifold $\pi$.

The case when $\Gamma_{1}^{p}=\mathrm{d} \Gamma^{p} \circ \rho_{1}, \Gamma_{2}^{p}=\mathrm{d} \Gamma^{p} \circ \rho_{2}, \Gamma_{12}^{p}=$ $\mathrm{d}^{2} \Gamma^{p}$ corresponds to a holonomic connection on the fibered manifold $\pi$.

The functions $\Gamma_{i}^{p}$ and $\Gamma_{i j}^{p}$ define nonholonomic, semiholonomic, or holonomic Ehresmann prolongation of a connection, respectively. 


\section{Material Connection}

Following the books $[1,2]$, the material body $\mathscr{B}$ is a trivial manifold without boundary. A coordinate chart $\kappa_{0}: \mathscr{B} \rightarrow$ $\mathbb{R}^{3}$ is identified as a reference configuration, a configuration of a material body $\mathscr{B}$ is an embedding

$$
\kappa: \mathscr{B} \longrightarrow \mathbb{E}^{3} \text {. }
$$

Choosing a frame in $\mathbb{E}^{3}$, we can identify $\mathbb{E}^{3}$ with $\mathbb{R}^{3}$. Now, one can associate with any given configuration $\kappa$ the deformation $\chi$ defined as the composition:

$$
\chi: \kappa \circ \kappa_{0}^{-1}
$$

In coordinates,

$$
x^{i}=\chi^{i}\left(X^{1}, X^{2}, X^{3}\right), \quad F_{I}^{i}=\frac{\partial x^{i}}{\partial X^{I}} .
$$

For a simple hyperelastic body, the constitutive equation is of the form: $\psi=\psi(F, X)$. Two points $X_{1}, X_{2} \in \mathscr{B}$ are materially isomorphic if there exists a non-singular linear map $P_{12}$, between their tangent spaces:

$$
P_{12}: T_{X_{1}} \mathscr{B} \longrightarrow T_{X_{2}} \mathscr{B}
$$

such that

$$
\psi\left(F P_{12}, X_{1}\right)=\psi\left(F, X_{2}\right)
$$

identically for all deformation gradients $F$. A body $\mathscr{B}$ is materially uniform if, and only if, there exists a material isomorphism $P(X)$ from a fixed point $X_{0} \in \mathscr{B}$ to each point $X \in \mathscr{B}$.

We shall call the point $X_{0}$ an archetypal material point, and the material isomorphisms $P(X)$ from the archetypal material point to the body points will be referred to as implants. A collection of such implants is a uniformity field.

A material archetype will be defined as a frame at $X_{0}$. We will say that two vectors at two different points $X_{1}$ and $X_{2}$ of an open set $U \subset \mathscr{B}$ are materially parallel with respect to the given uniformity field, if they have the same components in the respective local bases of the uniformity field.

A material symmetry at a point $X_{0} \in \mathscr{B}$ is material automorphism. A material symmetry $G$ at a point $X_{0}$ can be seen as a transformation such that

$$
\psi\left(F G, X_{0}\right)=\psi\left(F, X_{0}\right)
$$

The collection $\mathscr{G}_{0}$ of all material symmetries in $X_{0}$ constitutes group called material symmetry group.

In coordinates, let $E_{\alpha}$ be the natural basis of $\mathbb{R}^{3}$. By means of the uniformity maps $P(X)$ this basis induces a smooth field of bases in $U$, which we will denote by $p_{\alpha}(X)$. We now adopt a coordinate system in $U$, which we call $X^{I}$, with natural basis $e_{i}$; then

$$
p_{\alpha}=P_{\alpha}^{I} e_{I}
$$

The vector field $w$ can be expressed in terms of components in either basis, namely:

$$
w=\omega^{\alpha} p_{\alpha}=\omega^{I} e_{I} .
$$

Defining the Christoffel symbols of the local material parallelism as

$$
\Gamma_{I J}^{K}=P_{\alpha}^{K} \frac{\partial P_{I}^{-\alpha}}{\partial X^{J}}
$$

we can write material covariant derivative of the field $w$ wit respect to the given material parallelism

$$
\nabla_{I J}^{K}=\frac{\partial \omega^{K}}{\partial X^{J}}+\Gamma_{I J}^{K} \omega^{I}
$$

If the symmetry group $\mathscr{G}_{0}$ is trivial identity group, the material implants are unique. The local material connection is unique if the symmetry group $\mathscr{G}_{0}$ is discrete (i.e., consisting of a finite number of elements).

Recall, that the body is locally homogeneous if and only if there exists local material connection where Christoffel symbols are symmetric, for each point.

To apply the multiplication of connections on the material connections, we have to modify (12) for linear connections. The rest would be done by substitution of the previous characteristics in the equations. If two linear connections $\Gamma$ and $\bar{\Gamma}$ on the same base manifold $M$ are by coordinate formula (11), then $\bar{\Gamma} * \Gamma$ is given by (12). Should the connections $\bar{\Gamma}$ and $\Gamma$ be linear, the result would be obtained by substitution

$$
\begin{aligned}
& F_{i}^{p}=\Gamma_{i q}^{p} y^{q}, \\
& G_{i}^{p}=\bar{\Gamma}_{i q}^{p} y^{q}
\end{aligned}
$$

in (11), where $\Gamma_{i q}^{p}$ and $\bar{\Gamma}_{i q}^{p}$ are functions of the base manifold coordinates $x_{i}$. The equations of $\bar{\Gamma} * \Gamma$ would therefore look like

$$
\begin{gathered}
y_{i}^{p}=\Gamma_{i q}^{p} y^{q}, \quad y_{0 i}^{p}=\bar{\Gamma}_{i q}^{p} y^{q}, \\
y_{i j}^{p}=\frac{\partial \bar{\Gamma}_{i q}^{p}}{\partial x^{j}} y^{q}+\bar{\Gamma}_{i q}^{p} \Gamma_{j r}^{q} y^{r}+\bar{\Gamma}_{i q}^{p} y_{j}^{q} .
\end{gathered}
$$

Theorem 10. Let $\mathscr{B}$ be a material body and let $[\theta]$ be a class of second order connections. The constitutive equations are in the same projective class if and only if $[\theta]$ is semiholonomic.

Proof. The class $[\theta]$ of nonholonomic connections was introduced in Definition 7. If the element $\left(\Gamma_{1}, \Gamma_{2}, G\right)$ belongs to $[\theta]$, then from Theorem 6 the semiholonomity is equivalent to the property $\Gamma_{1}=\Gamma_{2}$. In particular, two constitutive equations determine two projectively equivalent connections of the first order.

Remark 11. The projective class of connections shares the same geodesics. In particular, if we describe "least energy deformation" of the material body based on two constitutive equations which lead to second order semiholonomic connection, then it is based on geodesics of one material connection of the first order. 
In fact, in our setting there is no extension of our result to connections of higher order than two (for explanation see [11]). This is the reason why the material equipped with two constitutive equations plays an interesting role in the theory of material bodies. Let us finally remark that the reformulation of the whole theory to the concept of infinitesimal connections on Lie groupoids can help; see [4].

\section{Conclusions}

We showed that if we represent the material properties by means of a second order connection, then its holonomity corresponds to the type of the material. Our ideas were motivated by handling materials with two constitution equations and it occurred that for more than two constitution equations a change of mathematical approach is needed.

\section{Acknowledgments}

The first author was supported by the Grant GA ČR, Grant no. 201/09/0981, and the second author by Grant no. FSI-S-11-3.

\section{References}

[1] M. Epstein and M. Elżanowski, Material Inhomogeneities and Their Evolution, Interaction of Mechanics and Mathematics, Springer, Berlin, Germany, 2007.

[2] M. Epstein, The Geometrical Language of Continuum Mechanics, Cambridge University Press, Cambridge, UK, 2010.

[3] I. Kolář, P. W. Michor, and J. Slovák, Natural Operations in Differential Geometry, Springer, Berlin, Germany, 1993.

[4] J. Virsik, "On the holonomity of higher order connections," Cahiers de Topologie et Geometrie Differentielle Categoriques, vol. 12, pp. 197-212, 1971.

[5] P. Vašík, "Transformations of semiholonomic 2- and 3-jets and semiholonomic prolongation of connections," Proceedings of the Estonian Academy of Sciences, vol. 59, no. 4, pp. 375-380, 2010.

[6] G. Atanasiu, V. Balan, N. Brînzei, and M. Rahula, Differential Geometric Structures: Tangent Bundles, Connections in Bundles, Exponential Law in the Jet Space, Librokom, Moscow, Russia, 2010.

[7] M. Rahula, P. Vašík, and N. Voicu, "Tangent structures: sectorforms, jets and connections," Journal of Physics: Conference Series, vol. 346, Article ID 012023, 2012.

[8] M. Doupovec and W. M. Mikulski, "Reduction theorems for principal and classical connections," Acta Mathematica Sinica, English Series, vol. 26, no. 1, pp. 169-184, 2010.

[9] P. Vašík, "On the Ehresmann prolongation," Annales Universitatis Mariae Curie-SkThlodowska A, vol. 61, pp. 145-153, 2007.

[10] M. Rahula, New Problems in Differential Geometry, vol. 8, World Scientific Publishing, River Edge, NJ, USA, 1993.

[11] W. M. Mikulski, "Natural transformations transforming functions and vector fields to functions on some natural bundles," Mathematica Bohemica, vol. 117, no. 2, pp. 217-223, 1992. 


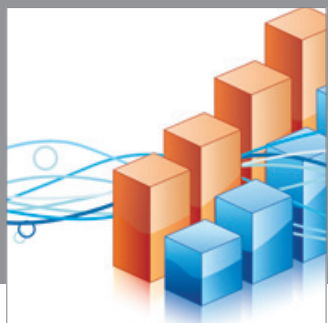

Advances in

Operations Research

mansans

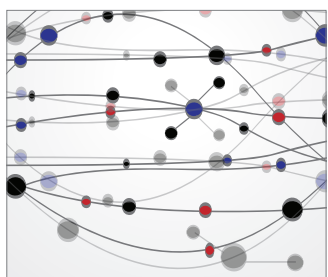

The Scientific World Journal
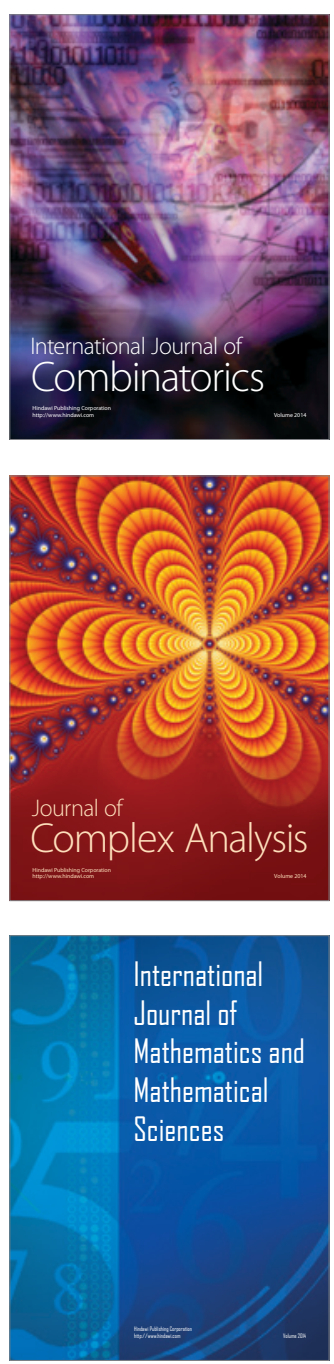
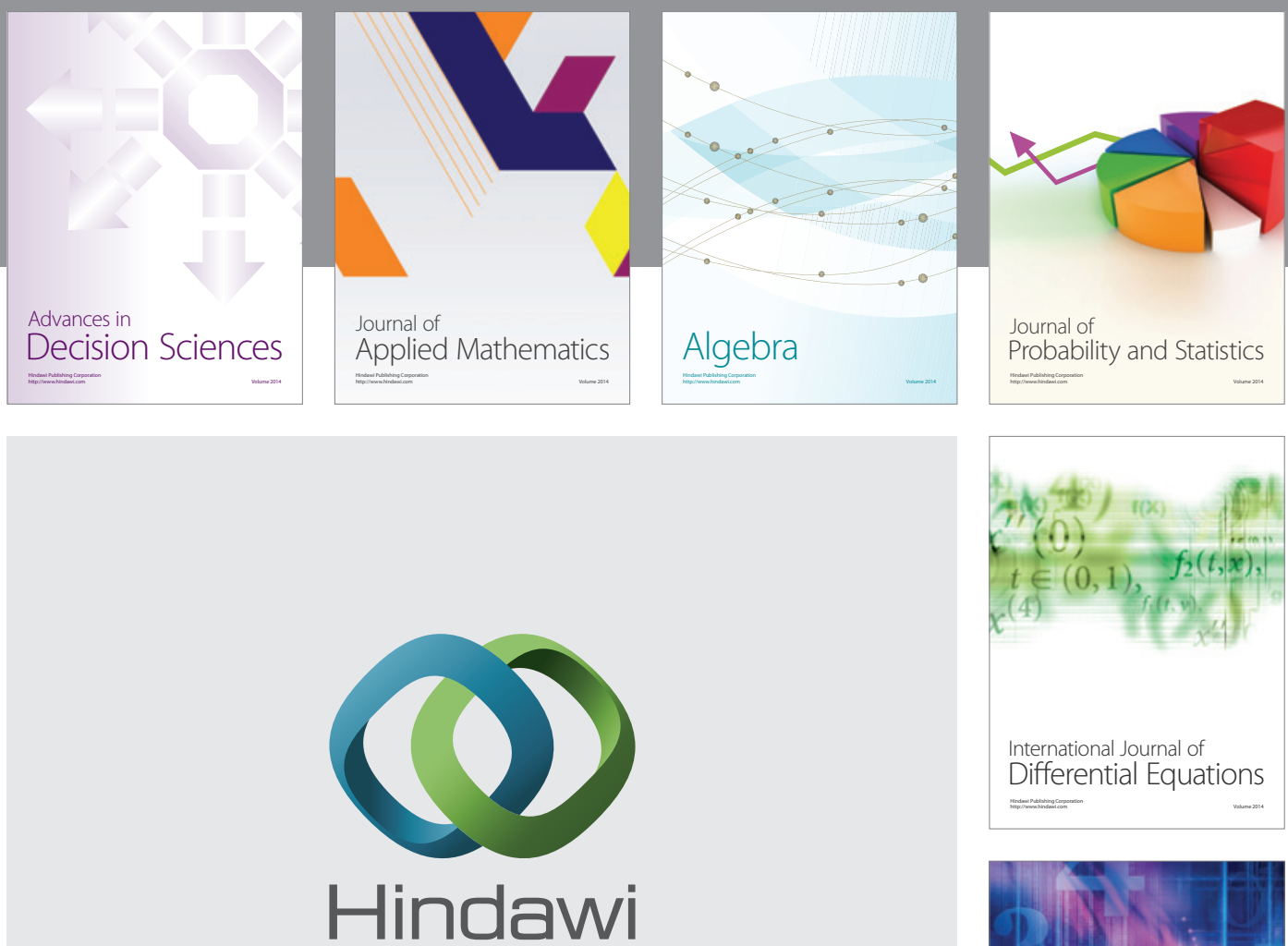

Submit your manuscripts at http://www.hindawi.com
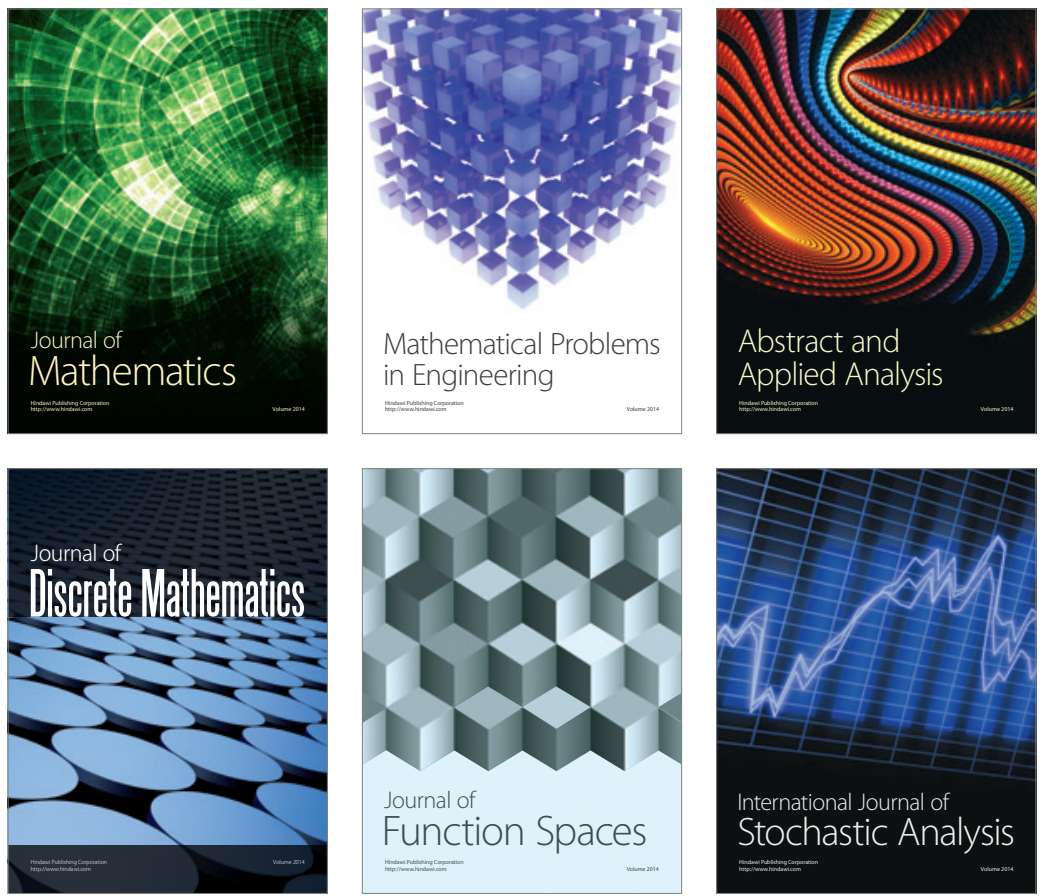

Journal of

Function Spaces

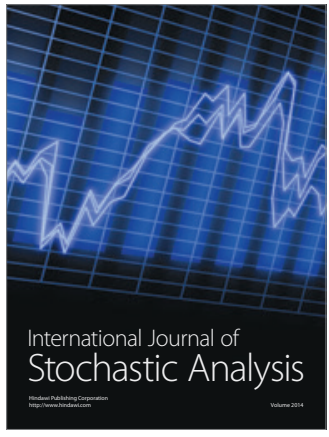

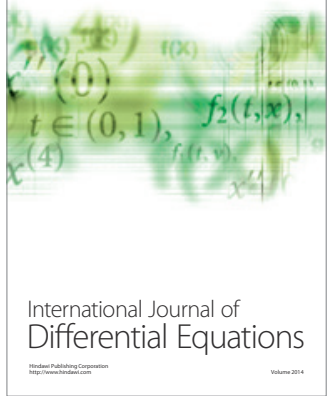
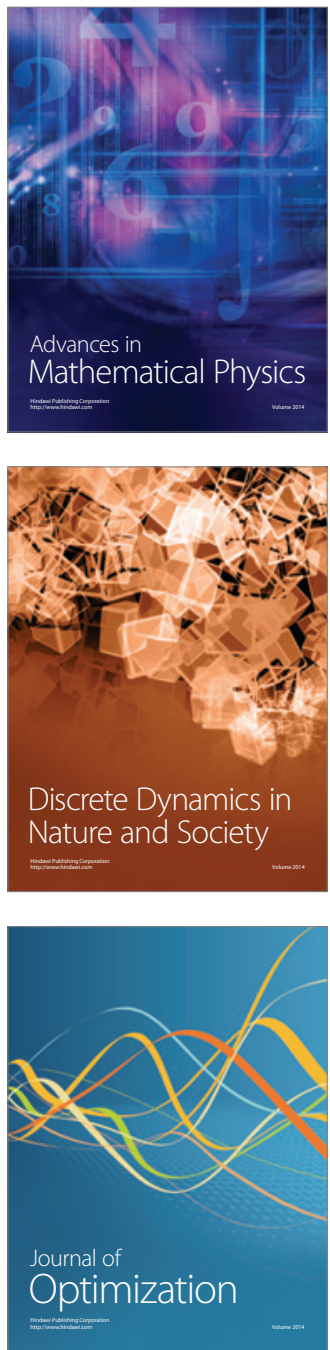